\title{
Abdominal wall movement in normals and patients with hemidiaphragmatic and bilateral diaphragmatic palsy
}

\author{
TIM HIGENBOTTAM, DAVE ALLEN, L. LOH, AND T. J. H. CLARK
}

From Guy's Hospital Respiratory Function Unit, and The Batten Unit, The National Hospital for Nervous Diseases, London, UK

Higenbottam, T., Allen, D., Loh, L., and Clark, T. J. H. (1977). Thorax, 32, 589-595. Abdominal wall movement in normals and patients with hemidiaphragmatic and bilateral diaphragmatic palsy. The abdomen and ribcage volume contribution to the tidal volume have been determined using magnetometers in three groups of subjects-normal males, patients with hemidiaphragmatic palsy, and patients with bilateral diaphragmatic palsy. In all three groups the main moving parts appear to be the anterior surfaces of the abdomen and ribcage even in the presence of bilateral diaphragmatic palsy.

In the group of patients with bilateral diaphragmatic paralysis the anterior abdominal wall moved inward paradoxically on inspiration. This 'negative' movement increased with larger tidal breaths and was best seen in recumbent patients. The normal subjects and those with hemidiaphragmatic palsy showed an outward movement of the anterior abdominal wall on inspiration which increased with larger tidal breaths. It is believed that the paradoxical movement of the abdomen in bilateral diaphragmatic palsy is a valuable clinical sign of this condition.

The contribution of the abdominal volume displacement to the tidal volume in the normal subjects and the hemidiaphragmatic palsy patients varied considerably between subjects and with different postures, but no difference could be discerned between the two groups. This supports the suggestion that the diaphragm is concerned more with maintaining a pressure difference between the abdomen and ribcage than displacing abdominal volume.

The diagnosis of complete or partial paralysis of the diaphragm can clinically pose a major difficulty. It has been suggested that the paradoxical inward movement of the abdomen, seen during inspiration in patients with bilateral diaphragmatic palsy, is a useful diagnostic sign (Campbell et al., 1970).

In order to clarify the value of this sign in patients with bilateral paralysis, and to assess its value in patients with hemidiaphragmatic palsy, three groups of subjects were studied-a normal group, a group with hemidiaphragmatic paralysis, and a group with bilateral paralysis. In each group the anteroposterior movement of the abdomen and ribcage was measured during breathing using two pairs of magnetometers (Mead et al., 1967). From these an estimate of the relative volume displacement of the abdomen and ribcage was made using the reported almost linear relationship between the change in anteroposterior diameters of each and their respective change in volume (Konno and Mead, 1967).

\section{Subjects, materials, and methods}

The three groups of subjects provided their informed consent and were made up of six normal male volunteers aged between 22 and 31 years; six patients with hemidiaphragmatic palsy, one female and five males between 22 and 45 years; and six patients with bilateral diaphragmatic palsy, three males and three females between 29 and 35 years.

The cause of the hemidiaphragmatic palsy was unknown in three patients, bronchial carcinoma in two, and secondary to juvenile poliomyelitis in one. The diagnosis was made by the presence of 
paradoxical movement of the affected hemidiaphragm on sniffing during radiographic chest screening, and the absence of neuromuscular activity was confirmed in each using external phrenic nerve stimulation (Newsom Davis, 1967), which was performed without prior knowledge of the affected side.

The diagnosis of bilateral diaphragmatic palsy had been made in an earlier study from the absence of or marked reduction in the transdiaphragmatic pressure during breathing (Newsom Davis et al., 1976). Three of these patients had acid maltase deficiency, one had familial spinal muscular atrophy, one had had earlier poliomyelitis, and in the last patient no cause was found for the diaphragmatic paralysis.

A lightweight Collins spirometer was used to measure the tidal volume during breathing in the normal subjects and in those with hemidiaphragmatic palsy, while the integrated flow signal from a Fleisch no. 2 pneumotachograph provided the tidal volume measurement in the patients with bilateral diaphragmatic palsy. In both cases, the spirometer, or the pneumotachograph, were calibrated before each person was studied, using a syringe pump.

The ribcage and abdomen anteroposterior movements during breathing were measured with two pairs of magnetometers (Mead et al., 1967) which were placed respectively at the level of the xiphisternum and the umbilicus, in the midline across the anteroposterior diameters. In addition to their individual outputs, an electrical adding circuit provided the sum of them both.

The four signals, tidal volume, ribcage, abdomen and their electrical sum, were recorded on a multichannel Mingograph recorder (Elema Schönlander).

In order to relate the anteroposterior displacement of the ribcage and abdomen to changes in tidal volume, use was made of the observation of Konno and Mead (1967) that, with the glottis closed, a change in volume of the ribcage is inversely related to an equal volume change of the abdomen. This has been called the isovolume manoeuvre (Fig. 1). Each subject is instructed to close his or her glottis close to end tidal expiration and slowly to transfer volume back and forth from the abdomen to the ribcage. By electrical scaling it is possible to render the outputs of each pair of magnetometers equal and in an opposite direction, so that the output of each pair of electrodes during breathing represents an equivalent change in volume. The subjects performed this isovolume manoeuvre before studying each posture.

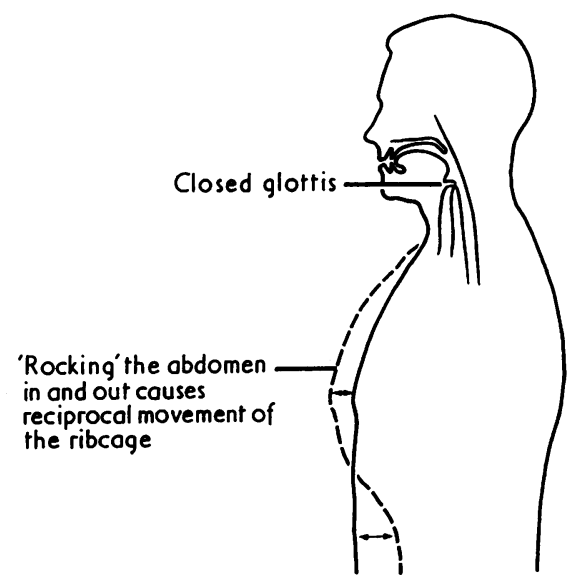

Fig. 1 The performance of the isovolume manoeuvre, which shows a subject with a closed glottis at end expiration volume slowly transferring volume back and forth between the abdomen and chest. The relative displacement of the anterior surfaces of the ribcage and abdomen are shown.

Each subject was studied where possible standing, sitting, and lying and was asked to breathe quietly for an interval while 17 to 26 breaths were

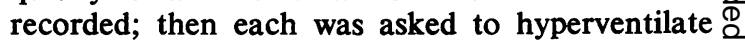
progressively. The respiratory volume was ob- $\overrightarrow{\vec{B}}$ tained from the tidal volume trace, and the $\frac{0}{3}$ direction and value of the inspiratory change in volume of the abdomen and ribcage was obtained from the magnetometer traces (Fig. 2).

\section{Results}

During quiet breathing in the erect and supine postures the normal subjects and those with the $\delta$ hemidiaphragmatic palsy exhibited a positive inspiratory volume (Fig. 2), that is, the abdomen 응 moved outwards on inspiration, while in the supine position all patients with bilateral diaphragmatic palsy showed a negative inspiratory volume (Fig. 3), the abdomen moving inwards on of inspiration. Four of these patients also showed this $\tilde{O}$ sign in the erect posture.

This difference in the direction of the inspiratory abdominal volume became more marked during the period of progressive hyperventilation, $\frac{\mathscr{D}}{\mathbb{D}}$ whereas normals and patients with hemidiaphrag- $\stackrel{\mathcal{D}}{?}$ matic palsy showed a progressively increasing $\square$ positive inspiratory volume with increases in tidal volume up to 2 litres (Fig. 4) in both supine and erect postures. Patients with bilateral diaphrag- $\mathbb{\otimes}$ matic palsy showed an increasing negative inspiratory volume in all postures (Fig. 5). 


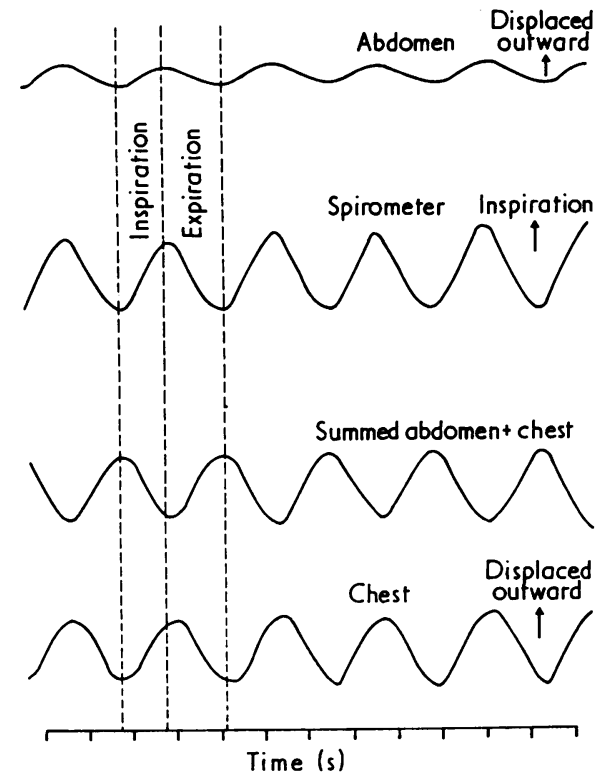

Fig. 2 A typical time-based recording obtained from the mingograph of a normal subject (N.O.) seated during quiet breathing. The abdomen inspiratory volume is illustrated on the top line beneath which is the tidal volume measurements, then the sum of the ribcage and abdomen volumes obtained from the electrical sum of the two pairs of magnetometers, and the bottom line represents the chest volume trace.

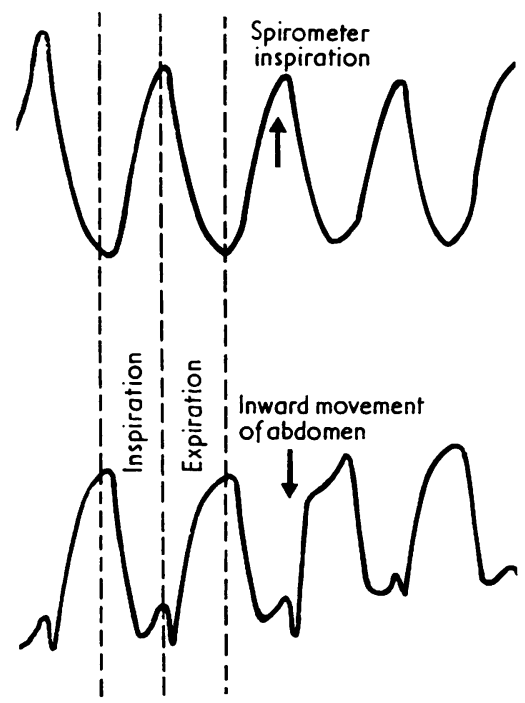

Fig. 3 The trace obtained from a typical mingograph recording of a patient (R.R., lying down) with bilateral diaphragmatic paralysis showing the change in volume of the abdomen (bottom line) compared with the tidal volume (upper line). A paradoxical reduction in volume of the abdomen during inspiration is shown.

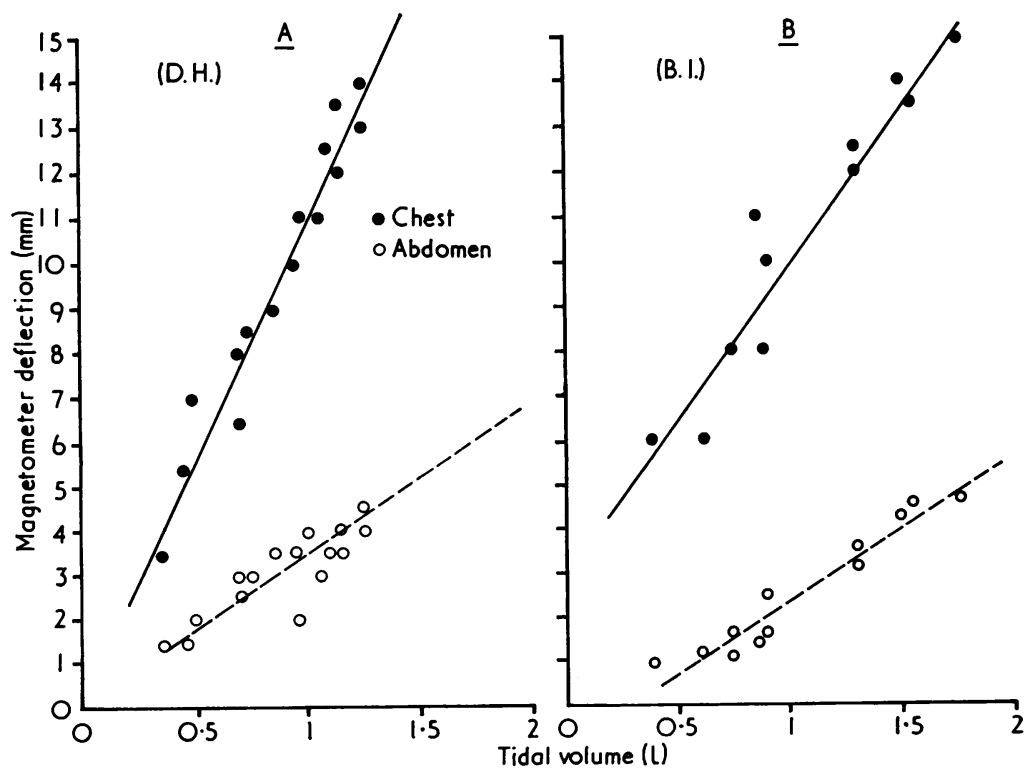

Fig. 4 Two scatter diagrams representing the typical relationship between the change in volume of the abdomen and the ribcage on the ' $y$ ' axis and the tidal volume on the ' $x$ ' axis. The plots were obtained during a period of progressive hyperventilation: $(A)$ a normal subject and $(B) a$ patient with hemidiaphragm palsy; both were sitting erect. 


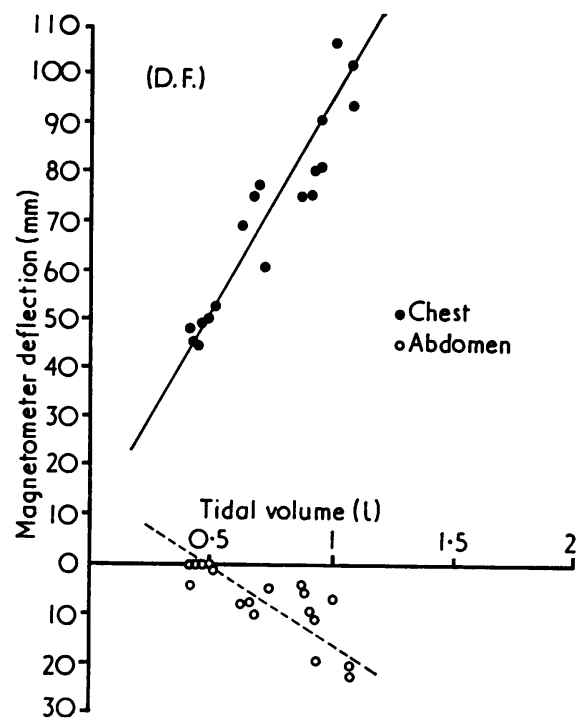

Fig. 5 A scatter diagram obtained from a patient sitting erect with bilateral diaphragmatic palsy during the period of hyperventilation: change in volume of the abdomen and the ribcage on the ' $y$ ' axis and the tidal volume on the ' $x$ ' axis. There is an increasing 'negative' abdominal inspiratory volume with increasing inspiratory tidal volumes.

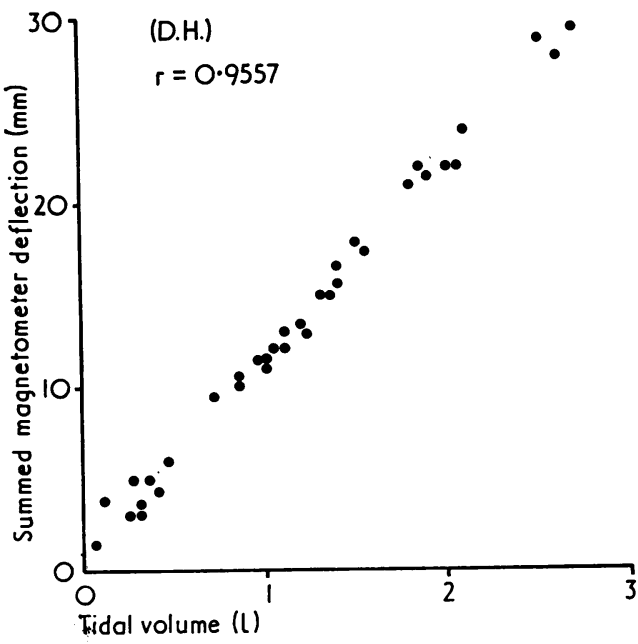

Fig. 6 A typical scatter diagram obtained using the measurements from a normal subject, sitting erect, during a series of consecutively increasing tidal volumes, showing on the ' $y$ ' axis the sum of abdominal and ribcage inspiratory volumes with the tidal volume on the ' $x$ ' axis.

Table Correlation coefficients $(r)$ for all subjects in each posture between the sum of the abdominal and ribcage inspiratory volumes estimated from the magnetometers and the tidal volume measurement over a range of tidal volumes

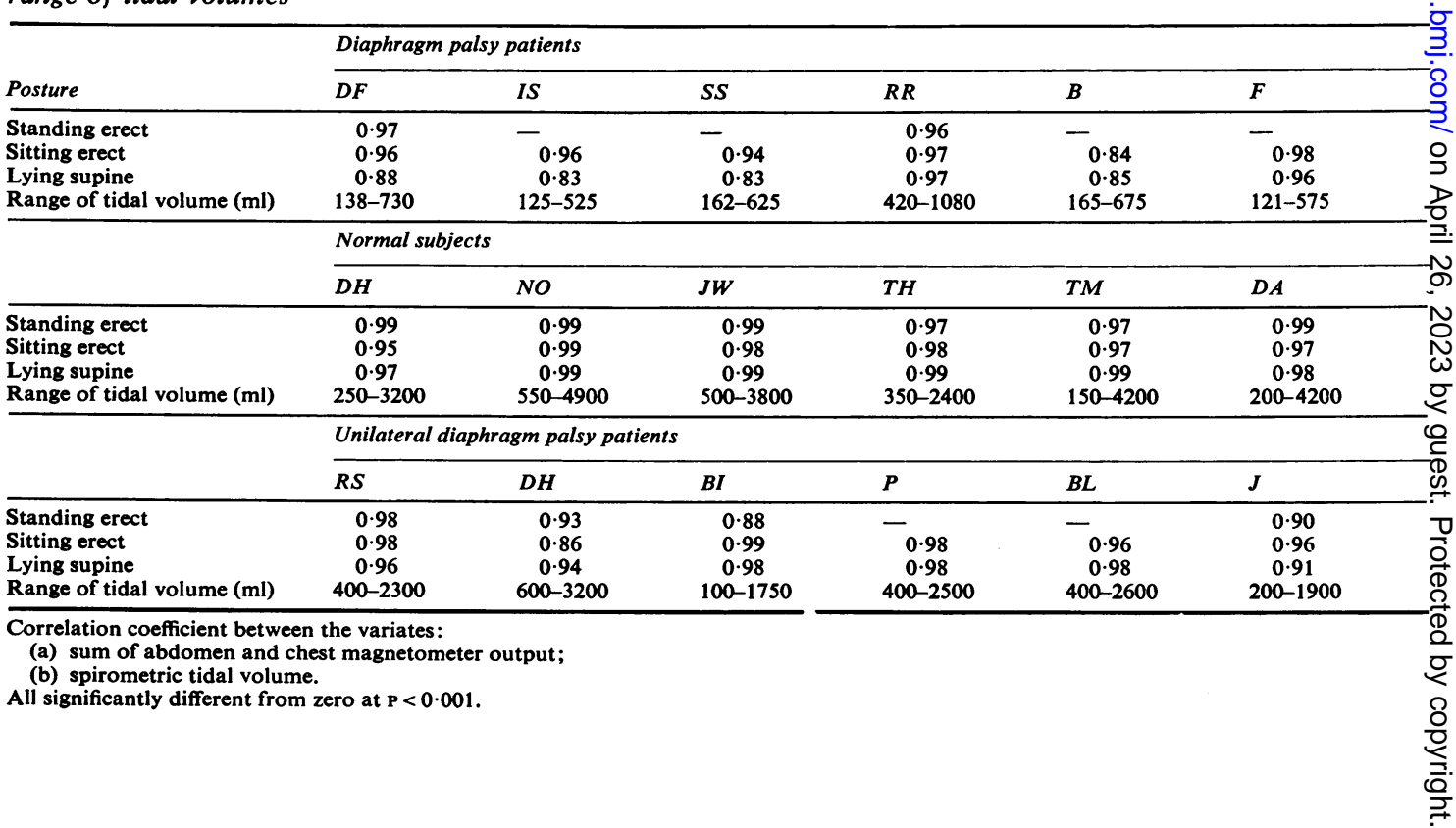



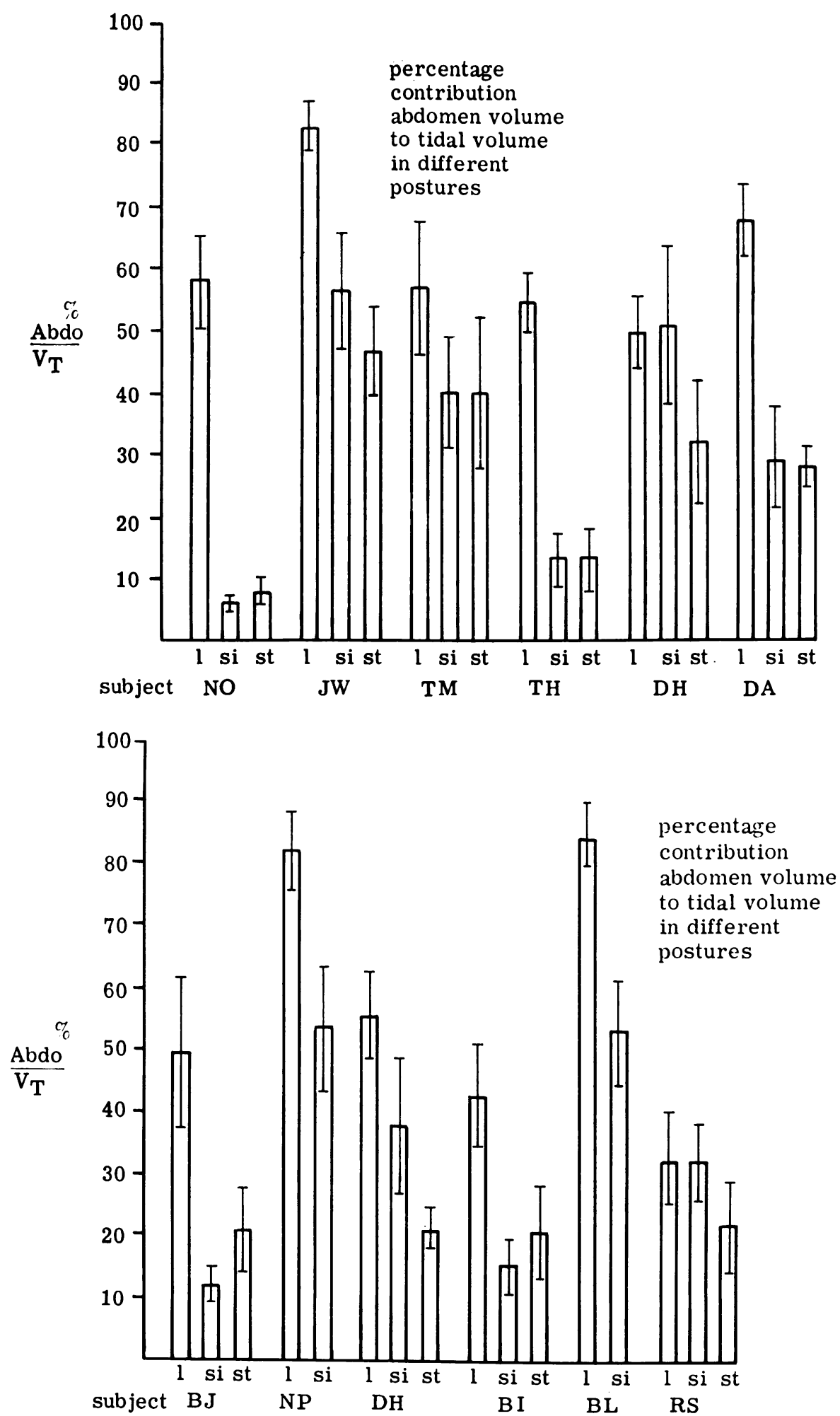

Fig. 7 Two sets of histograms for the normal subjects (above), and the patients with hemidiaphragmatic palsy (below). The height of each histogram block represents the percentage contribution of the change in volume of the abdominal inspiratory volume to the total ribcage and abdomen inspiratory volume. There is a block for each posture, ' $l$ ' for lying, 'si' for sitting, and 'st' for standing, for each subject. The mean of 17-28 breaths was used to obtain each mean value which were measured during a quiet breathing period when the tidal volume range for all the subjects was between 400 and $1000 \mathrm{ml}$ (the line and bar represent two standard deviations). 
During the period of progressive hyperventilation there was a high correlation between the electrical sum of the magnetometer outputs of the ribcage and abdomen and the tidal volume in all the subjects irrespective of whether they had diaphragmatic palsy or not (Table). The linearity of this relationship was shown on scatter diagrams (Fig. 6).

The proportion that the abdominal inspiratory volume contributed to the sum of the ribcage and abdomen inspiratory volumes during quiet breathing varied enormously between subjects and according to posture, but no difference could be seen when comparing the normal subjects with those with hemidiaphragmatic palsy (Fig. 7).

\section{Discussion}

The value of the paradoxical sucking inwards of the abdomen during inspiration as a diagnostic sign of bilateral diaphragmatic palsy is emphasised. Differences in age and sex of the groups would be unlikely to contribute to these findings; Sharp and others (1975) found little difference in the abdominal volume contribution to inspiration in the different age groups and between sexes. Patients are best studied while supine, and the sign is augmented by hyperventilation. This sign was not seen when only one diaphragm was paralysed.

The high correlation between the sum of the ribcage and abdominal inspiratory volumes, as estimated by the magnetometers, and the tidal volume supports the finding of Konno and Mead (1967) that the respiratory system has only two main moving parts, the anterior walls of the abdomen and the ribcage. It also provides support for the use of these magnetometers as an indirect method of estimating the tidal volume.

The enormous inter-subject variation in the proportion that the abdominal inspiratory volume contributes to the tidal volume during quiet breathing prevents any quantitative comparison between the normal group and the patients with hemidiaphragmatic palsy. This result and the failure to observe paradoxical movement of the abdomen during inspiration leads us to conclude that the assessment of anterior abdominal movement is unhelpful in the diagnosis of hemidiaphragmatic paralysis.

The large inter-subject variation seen in Fig. 7 fails to substantiate the view of Konno and Mead that the proportion of abdominal inspiratory volume contribution to the tidal volume is solely determined by the percentage that the active dia- phragm contributes to the total external lung surface area. They suggest that this should be $28 \%$ (Konno and Mead, 1967), based on Keith's (1909) estimates of diaphragmatic surface area in man. Our finding that the abdominal inspiratory volume contributed between 10 and $90 \%$ of the tidal volume in both the normal subjects and those with o hemidiaphragmatic palsy would suggest that the $\vec{\circ}$ role of the diaphragm is concerned less with $\overrightarrow{\vec{\omega}}$ volume displacement of the abdomen than with maintaining a pressure difference between the $\overrightarrow{\overrightarrow{2}}$ relatively rigid ribcage and the collapsible abdomen. In support of this, when changing from the erect to supine posture, generally the normal sub- of jects and those with hemidiaphragmatic palsy 0 showed an increased abdominal inspiratory volume 0 contribution to the tidal volume. This has been explained solely in the terms of a change in the relative compliances of the ribcage and abdomen with posture (Nisell, 1963) in that the ribcage is less compliant and the abdomen more compliant when the supine posture is compared with the $\overrightarrow{0}$ erect posture. This role of the diaphragm, al- V though maintained in hemidiaphragmatic palsy, is lost when both sides of the diaphragm are paralysed, which explains the indrawing of the anterior abdominal wall during inspiration, particularly in the supine posture.

We thank Dr. J. Newsom Davis, of the National Hospital for Nervous Diseases, and Dr. G. W. Scott, of Guy's Hospital, for permission to study their patients. We acknowledge the help and criticism in the preparation of this paper of Dr. M. Goldman.

\section{References}

Campbell, E. J. M., Agostoni, E., and Newsom Davis, J. (1970). The Respiratory Muscle; Mechanics and $\mathrm{O}$ Neural Control, 2nd edition. Lloyd-Luke, London.

Keith, Sir Arthur (1909). The Mechanism of Respira- $\frac{D}{0}$ tion in Man. In Further Advances in Physiology, edited by L. E. Hill, pp. 182-207. Edward Arnold, N London.

Konno, K., and Mead, J. (1967). Measurement of the $\mathrm{N}$ separate volume changes of rib cage and abdomen $N$ during breathing. Journal of Applied Physiology, 22, 407-422.

Mead, Jere, Peterson, N., Grimby, G., and Mead, Judson (1967). Pulmonary ventilation measured \&

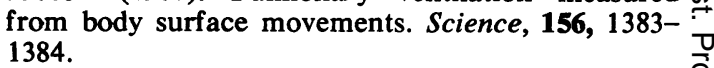

Newsom Davis, J. (1967). Phrenic nerve conduction in $\frac{\vec{D}}{\mathbb{D}}$ man. Journal of Neurology, Neurosurgery, and Psychiatry, 30, 420-426.

Newsom, Davis, J., Goldman, M., Loh, L., and $\frac{\Omega}{\sigma}$ Casson, M. (1976). Diaphragm function and alveolar 
hypoventilation. Quarterly Journal of Medicine, 45, 87-100.

Nisell, O. (1963). Mechanics of abdomen and thorax in man during natural breathing. Acta Physiologica Scandinavica, 59, 85-96.

Sharp, J. T., Goldberg, N. B., Druz, W. S., and Danon, J. (1975). Relative contributions of rib cage and abdomen to breathing in normal subjects. Journal of Applied Physiology, 39, 608-618.

Requests for reprints to: Dr. T. Higenbottam, Clinical Science Laboratories, Guy's Tower, Guy's Hospital, London Bridge, SE1 9RT, UK. 